\title{
Light-Scattering Patterns from Polyethylene Films in Relation to Spherulitic Crystalline Texture*
}

\author{
Masahiko MotegI, ${ }^{* *}$ Takashi OdA,*** Masahiko MoRItANI, and Hiromichi KAwAI \\ Department of Polymer Chemistry, Faculty of Engineering, Kyoto University, Kyoto, Japan.
}

(Received August 13, 1969)

\begin{abstract}
H_{v}$ light-scattering patterns from film specimens of relatively low density polyethylenes differ from the so-called "four leaf clover pattern" which is often found in the case of a well-grown spherulitic texture of polyethylene and can be derived from Stein's formulation of the scattered pattern. The pattern is some modifications removed from the "four leaf clover" pattern so as to have a rather long leafstalk, and may be called "four tennis rackets" arranged orthogonally.

Some modifications of Stein's formulation for an isolated spherulite of a rather simple and regular anisotropy are proposed by taking the distribution of the size of spherulites into account, taking the spherulite anisotropy, $\left(\alpha_{r}-\alpha_{t}\right)$, as a function of distance from the center of the spherulite, and further making the boundary of the spherulite less clear.

The former two modifications do not give any essential change in the scattering pattern from that predicted by the Stein's formulation, except for some shifts of the scattering angle at maximum intensity, while the latter modification gives the extension of the logarithmic contour plot of scattered intensity to lower scattering angles, and confirms the pattern of the "four tennis rackets."

In addition, a modification of the spherulitic crystalline texture to a sheaf-like texture by using a two-dimensional symmetric sector model also gives an extension of the logarithmic contour plot to lower scattering angles, even showing the strongest scattered intensity at the lower angles when the sector angle is taken as less than $50^{\circ}$.
\end{abstract}

KEY WORDS Light-Scattering / $\boldsymbol{H}_{v}$ Pattern / Polyethylene Film /

Spherulite / Anisotropy / Size / Boundary / Calculation /

Theoretical studies of light-scattering from polymeric solids for isotropic but heterogeneous systems were first carried out by Debye-Bueche ${ }^{1}$ in 1949 and were extended to anisotropic systems by Stein and co-workers using a polarized light-scattering technique for crystalline polymers having crystalline superstructure. Two types of approach, i.e., the amplitude method applicable to the system having a superstructure of high symmetry ${ }^{2}$, and the correlation method for a system of low

* Part of the M.S. thesis of M. Motegi presented at the Department of Polymer Chemistry, Faculty of Engineering, Kyoto University, on March 11th, 1969; presented before the 18th Annual Meeting of the Society of Polymer Science, Japan, Kyoto Conference Hall, Kyoto, on May 20th, 1969.

** Present address: Film Laboratory, Toyo Rayon Co., Ltd., Ohtsu, Shiga, Japan.

*** Present address: Polymer Research Institute, University of Massachusetts, Amherst, Massachusetts 01002 U.S.A. symmetry $^{3,4}$, have been proposed.

Within the two approaches, light-scattering from the spherulitic texture of crystalline polymers has been successfully explained by means of the amplitude method. ${ }^{2}$ However, light-scattering patterns from particular crystalline polymers such as some kinds of polyethylenes, do not necessarily agree in detail with the pattern predicted from the theory, as will be illustrated later, and probably reflect considerable difference of spherulitic texture among the polyethylenes.

In this paper, some modifications of Stein's formulation for polarized light-scattering from spherulitic texture will be proposed by changing to some extent the structural parameters so as to reflect the difference of the spherulitic texture.

TEST SPECIMENS AND

EXPERIMENTAL PROCEDURES AND RESULTS

Table I shows physical characterizations of 
Table I. Physical characterizations of test polymers, casting conditions and some physical indices of cast films

\begin{tabular}{|c|c|c|c|c|c|c|c|}
\hline \multicolumn{2}{|c|}{$\begin{array}{l}\text { Specimen } \\
\text { cord }\end{array}$} & \multirow{2}{*}{$\begin{array}{c}\text { Melt Index } \\
\text { ca. } 4\end{array}$} & \multirow{2}{*}{$\frac{\bar{M}_{w}^{\mathrm{b}}}{33000}$} & \multirow{2}{*}{$\begin{array}{c}\begin{array}{c}\text { Degree of c } \\
\text { branching }\end{array} \\
2.7\end{array}$} & \multirow{2}{*}{$\begin{array}{l}\text { Casting condition } \\
\begin{array}{l}\text { Molten at } 150^{\circ} \mathrm{C} \text { and } \\
\text { cooled gradually } \\
\text { down to room tem- } \\
\text { perature. }\end{array}\end{array}$} & \multirow{2}{*}{$\begin{array}{c}\begin{array}{c}\text { Density } \\
(\mathrm{g} / \mathrm{cc})\end{array} \\
0.918\end{array}$} & \multirow{2}{*}{$\begin{array}{c}\begin{array}{c}\text { Radius of } \\
\text { spherulite } \\
(\mu)\end{array} \\
1.80\end{array}$} \\
\hline YK & 3200 & & & & & & \\
\hline YK & 60 & ca. 4 & 33000 & 2.3 & ibid. & 0.930 & 3.15 \\
\hline YK & 50 & ca. 4 & 33000 & 2.6 & ibid. & 0.926 & 2.80 \\
\hline YK & 30 & ca. 4 & 33000 & 2.7 & ibid. & 0.922 & 1.85 \\
\hline $\mathrm{KP}$ & 119 & 2.0 & 33000 & 1.3 & ibid. & 0.934 & 6.65 \\
\hline DFD & 4164 & ca. 2 & 34000 & 1.6 & ibid. & 0.930 & 2.60 \\
\hline KR & 1051 & - & - & 1.5 & ibid. & 0.934 & 3.10 \\
\hline G & 201 & 2.0 & 42000 & 2.5 & ibid. & 0.923 & 3.75 \\
\hline L & 402 & 3.5 & 37000 & 2.4 & ibid. & 0.925 & 3.95 \\
\hline A & 4002 & 0.42 & 47000 & 2.2 & ibid. & 0.924 & 2.15 \\
\hline
\end{tabular}

a ASTM D-1238-57T.

b Calculated from intrinsic viscosity in xylene solution at $75^{\circ} \mathrm{C}$ by using the Harris equation. ${ }^{5}$

c Number of $\mathrm{CH}_{3} / 100$ carbon atoms, determined from infrared spectroscopy at 1376 and $1368 \mathrm{~cm}^{-1}$ at melt.

d Measured by floatation method in mixture of benzene and carbon tetrachloride at $25.0^{\circ} \mathrm{C}$.

e Measured from $H_{v}$ light-scattering pattern by using Eq. 3 .

about ten kinds of polyethylenes used as test polymers together with some physical indices of bulk polymers cast from the molten state of test polymers into a film shape of about $0.1 \mathrm{~mm}$. thickness by using a laboratory press. As seen in the table, the polyethylenes are classified as low to medium density polyethylenes having density values ranging from 0.918 to 0.934 at $25.0^{\circ} \mathrm{C}$.

Figure 1 shows a plot of the degree of crystallinity determined by X-ray diffraction ${ }^{6}$ against that determined from the density of bulk polymer by assuming the specimen to be a bi-phase system of

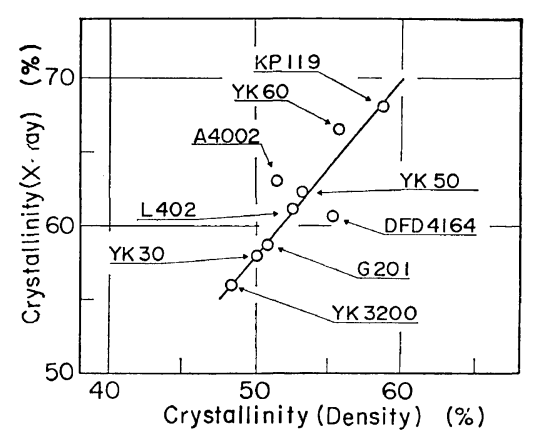

Figure 1. Relationship between the degree of crystallinity determined from X-ray diffraction and that from bulk density for the film specimens of various kinds of low and medium density polyethylenes. crystalline and noncrystalline components of respective densities of 1.000 and $0.855 .^{7}$ As seen in the figure, the plot is represented by a fairly good linear relationship irrespective of the kinds of polyethylenes, although the slope of the linear relationship is somewhat larger than $45^{\circ}$.

Figures 2 and 3 are plots of the radius of spherulite determined from the light-scattering pattern ${ }^{2}$ of each film specimen against the density of bulk polymer and the sonic modulus at $10 \mathrm{kc}$, respectively. As seen in the figures, there are two

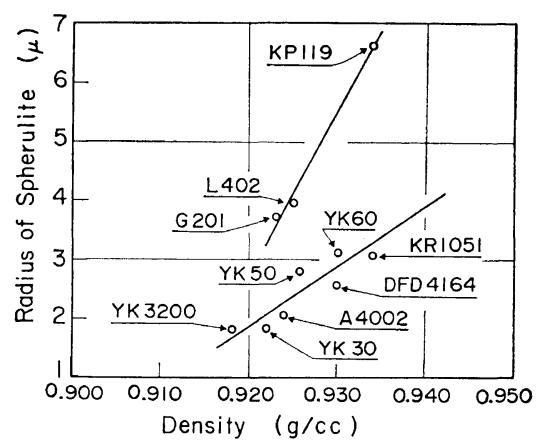

Figure 2. Relationship between the radius of the spherulite determined from $H_{v}$ light-scattering pattern and bulk density for the film specimens of various kinds of low and medium density polyethylenes. 


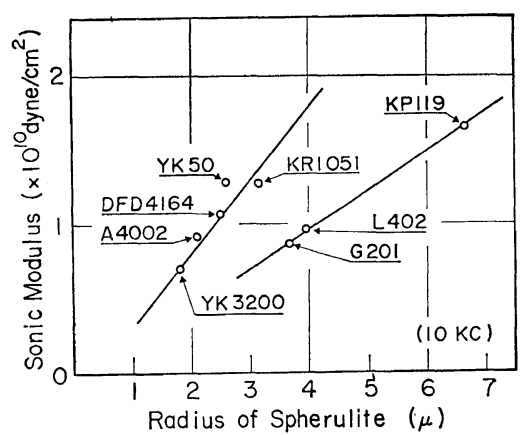

Figure 3. Relationship between the radius of the spherulite determined from $\boldsymbol{H}_{v}$ light-scattering pattern and sonic modulus for the film specimens of various kinds of low and medium density polyethylenes.

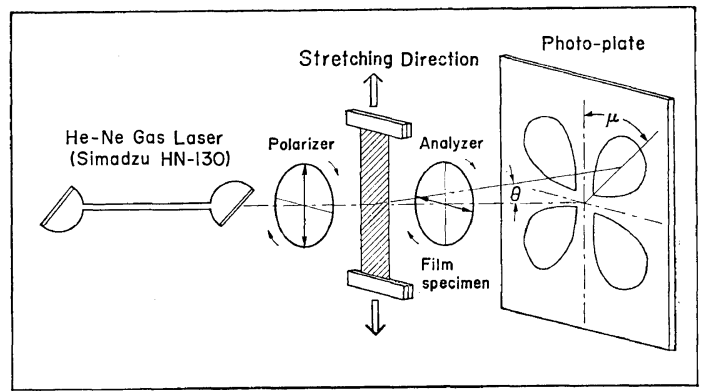

Figure 4. Schematic diagram of photographic lightscattering apparatus used in this experiment.

groups of polyethylenes plotted by different linear relationships; i.e., G 201, L 402 and KP 119 belong to one group and the others to the other group.

Figure 4 shows a schematic diagram of a photographic light-scattering apparatus used in this experiment. The film specimen and the photographic plate are placed normal to the incident beam, which is radiated from a $\mathrm{He}-\mathrm{Ne}$ gas laser apparatus, Model NH-130 Shimadzu Seisakusho. The incident beam is well-collimated and polarized to any direction within a plane perpendicular to the beam itself by using a polarization rotator, Model 310, Spectra Physics Inc., and also monochromized to have a wavelength of $6328 \AA$. An analyzer is placed between the film specimen and the photographic plate, whose polarization direction is also rotatable within a plane perpendicular to the incident beam so that any combination of polarization scattering can be performed by changing the polarization directions of the polarizer and analyzer with respect to the stretching direction of the film specimen.

Figure 5 shows the $H_{v}$ light-scattering patterns from several kinds of polyethylene film specimens, where the polarization direction of the polarizer is kept vertical and that of the analyzer horizontal. As seen in the figure, the shape of the scattering pattern is again classified into the two groups; G 201 and L 402, and the remainder. For the former group, the pattern is the so-called "four leaf clover pattern" which is quite close to the pattern predicted from Stein's formulation, ${ }^{2}$ as will be discussed later, while for the latter group the clover pattern is modified so as to have a rather long leafstalk resembling "four tennis rackets" arranged orthogonally. The facts that the scattering patterns from the former group are, in general, concentrated in lower scattering angles, sharper at the boundary of the pattern, and closer to Stein's formulation than those from the latter group, suggest that the spherulites for the former group are larger in size, more perfect in crystalline super-structure, and closer to the regular structure as postulated in Stein's model for the formulation.

Figures 6 and 7 show the changes of the $H_{v}$ scattering patterns with $\%$-elongations of uniaxial stretching and residual \%-elongations after releasing the stretched specimens of the corresponding $\%$-elongations (left-hand side) for two representatives of the two groups, G 201 and YK 3200, respectively. As recognized from the figures, the scattering pattern is elongated to the horizontal direction with increasing of the \%-elongation or residual $\%$-elongation, while the pattern after releasing is, in general, much sharper than that during stretching, probably reflecting the recovery of regularity of the super-structure. However, the change of the scattering pattern with the \%elongations differs considerably within the two specimens; i.e., for $G 201$ the change of the scattering pattern follows that predicted from Stein's formulation ${ }^{8}$ or from the Samuels formulation, ${ }^{9}$ but for YK 3200 the change of the scattering pattern deviates considerably from that predicted from the formulations, even suggesting the existence of two types of spherulites differing in compliance for deformation. 
$H_{V}$ Scattering from Polyethylenes (unstretched)

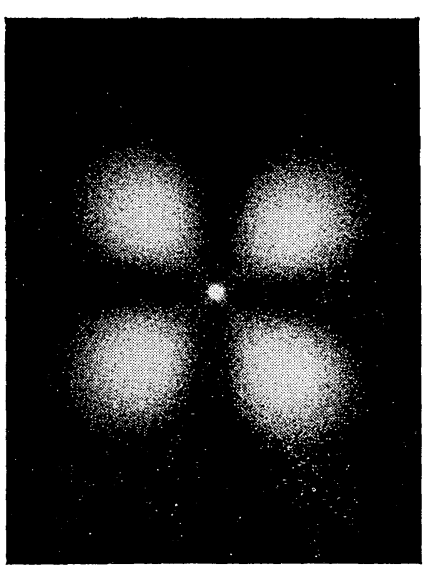

A 4002

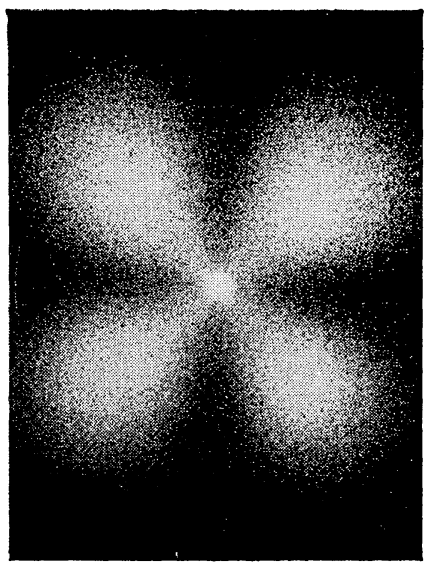

YK 3200

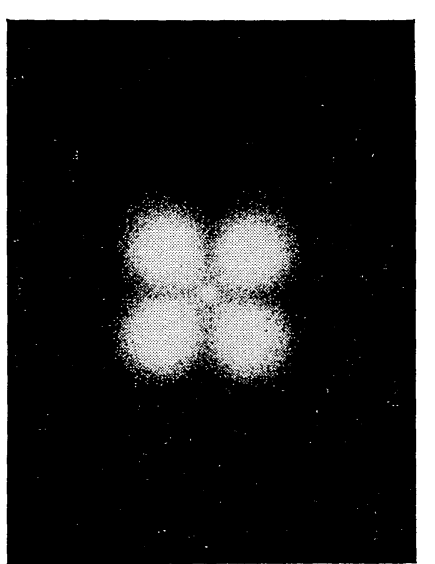

G201

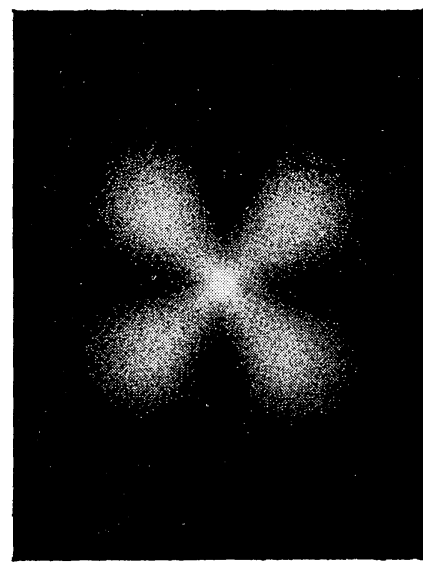

DFD 4164

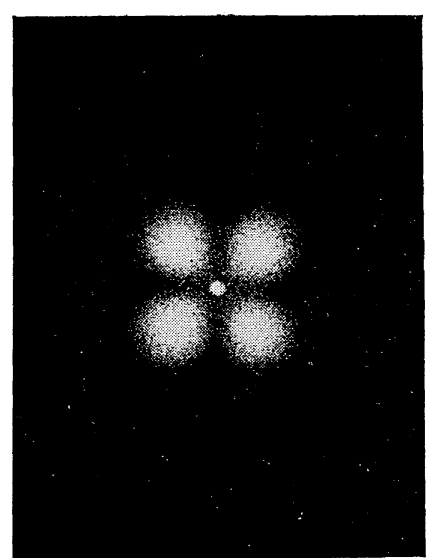

L 402

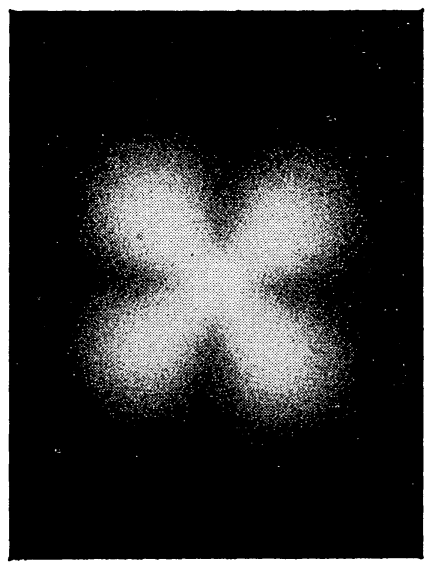

KRIO5I

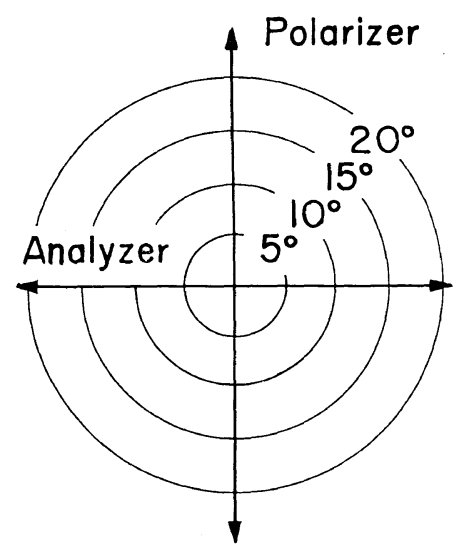

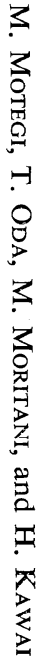

Figure 5. $H_{v}$ light-scattering patterns from film specimens of various kinds of low and medium density polyethylenes. 
Light-Scattering from Polyethylene Spherulite

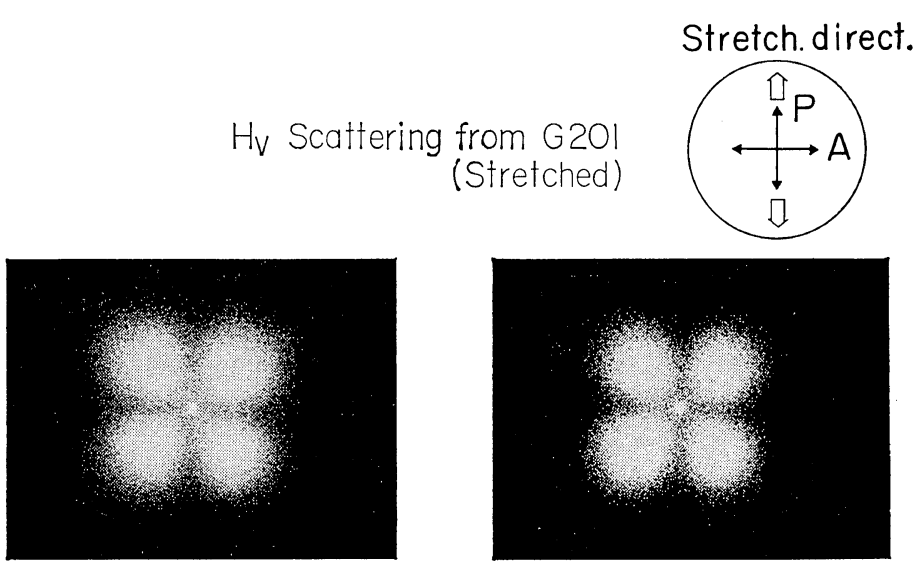

$5 \%$-elongation

$3 \%$-residual strain

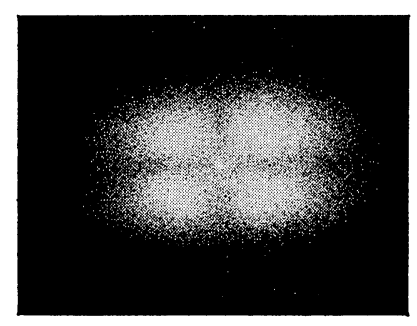

$30 \%$

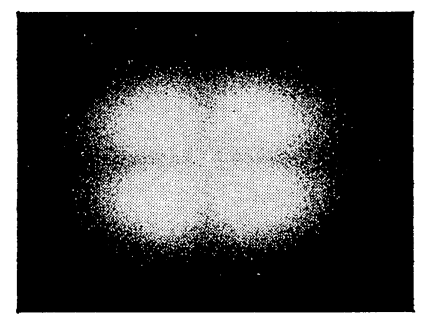

$10 \%$

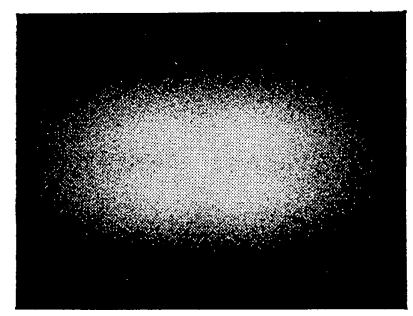

$50 \%$

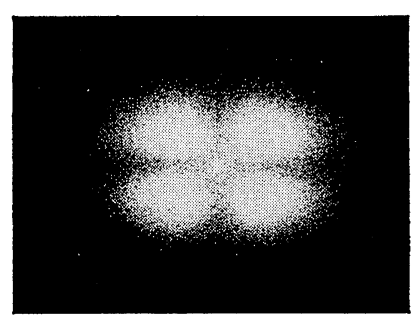

$16 \%$
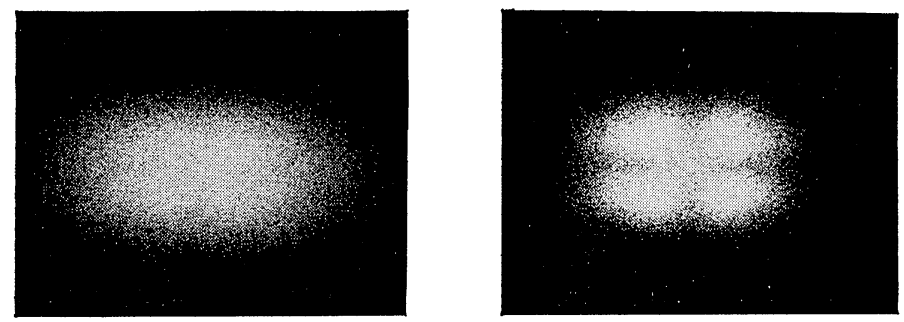

$68 \%$

(Stretched)

(Released)

Figure 6. The changes of $H_{v}$ light-scattering patterns with \%-elongations during uniaxial stretching of film specimen (G 201) and with residual \%-elongations after releasing the stretched states of corresponding $\%$-elongations (left-hand side). 


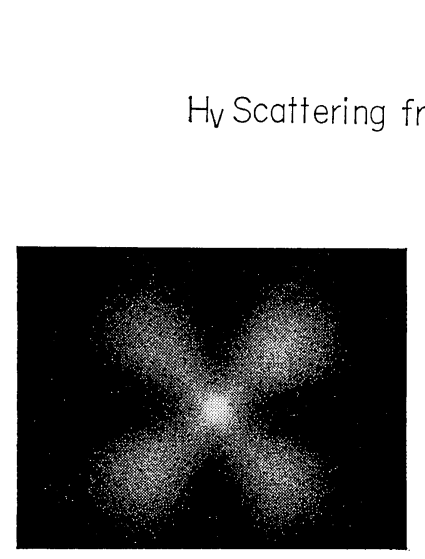

$8 \%$-elongation

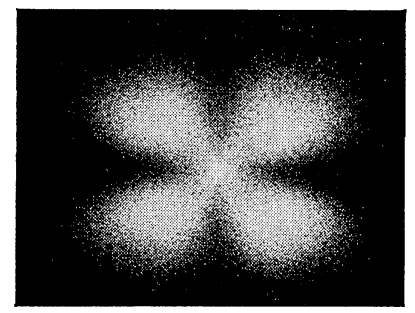

$15 \%$

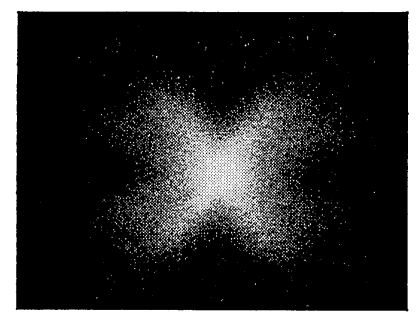

$34 \%$

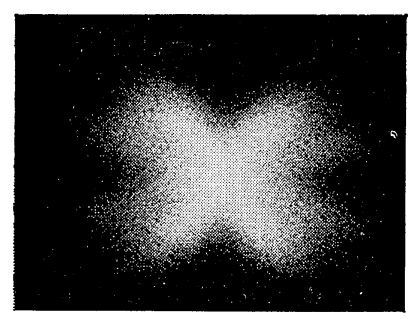

$55 \%$

(Stretched)

Stretch. direct.
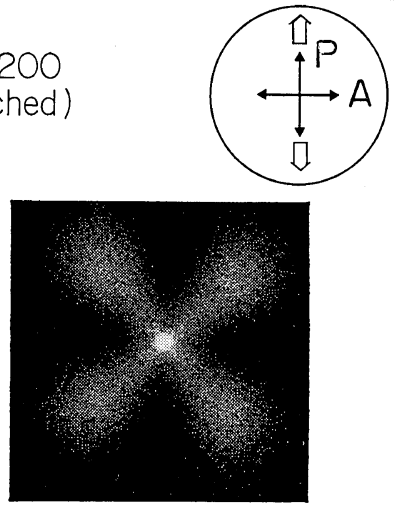

$2 \%$-residual strain

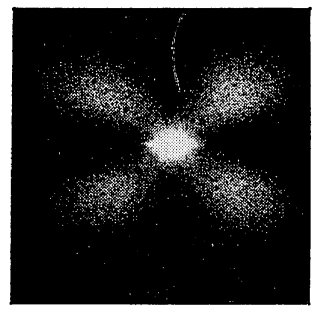

$7 \%$

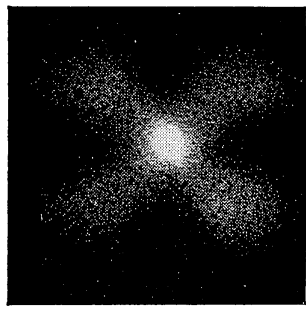

$10 \%$

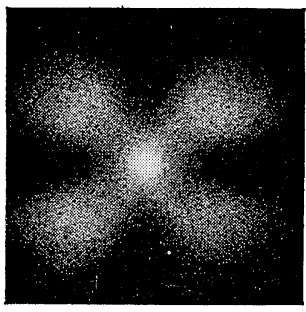

$17 \%$

(Released)

Figure 7. The changes of $H_{v}$ light-scattering patterns with \%-elongations during uniaxial stretching of film specimen (YK 3200) and with residual \%-elongations after releasing the stretched states of corresponding $\%$-elongations (left-hand side). 

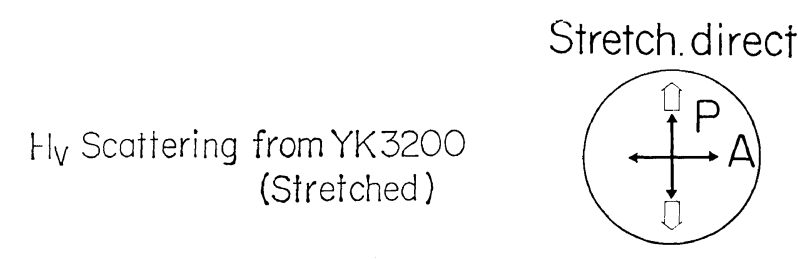

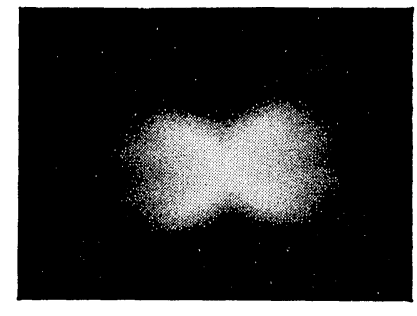

$75 \%$-elongation

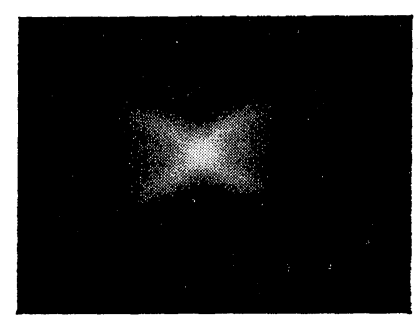

$100 \%$

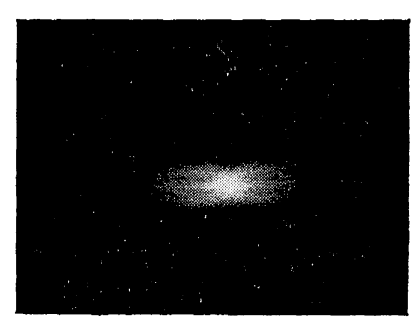

$200 \%$

(Stretched)

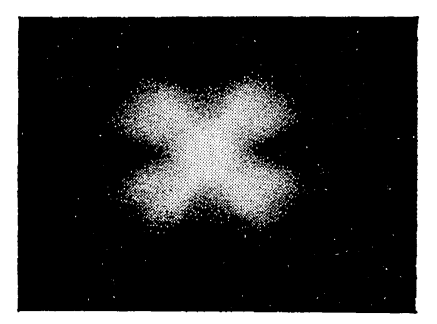

$26 \%$-residual strain

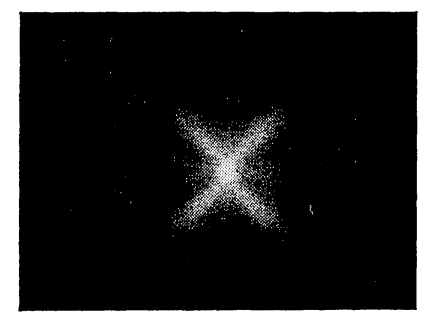

$46 \%$

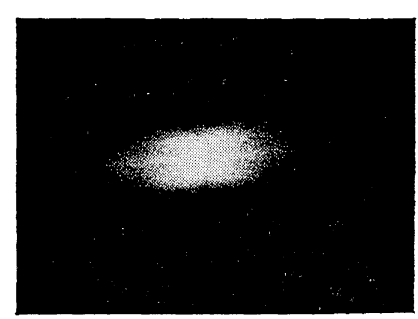

$153 \%$

(Released)

Figure 8. The changes of $H_{v}$ light-scattering patterns with $\%$-elongations during uniaxial stretching of film specimen (YK 3200) and with residual \%-elongations after releasing the stretched states of corresponding $\%$-elongations. (Continued from Figure 7) 
This is more clearly shown in Figure 8 for higher $\%$-elongations up to $100 \%$, where the scattering intensity as well as the scattering pattern itself degenerate, even giving a horizontal streak which suggests the disintegration of at least one type of spherulites of larger compliance.

The above differences in the physical indices and the polarized light-scattering patterns found among the two groups of the polyethylene films may be ascribed to the differences of the regularity and perfectness of the crystalline super-structures and the crystal structures themselves. In the following calculations, some modifications of Stein's formulation, which were based on a rather simple and regular morphological model of polyethylene spherulite, will be proposed by changing to some extent structural parameters in order to realize, at least, the polarized lightscattering pattern of "four tennis rackets" found for the undeformed films of polyethylenes, such as YK 3200 and DFD 4164, in Figure 5.

\section{CALCULATIONS}

The amplitude of scattered light from a spherulite, as shown in Figure 9, is given by

$$
E=A \int_{v}(M \cdot o) \cos [k(r \cdot s)] d \tau
$$

where $A$ is a constant, $M$ is an induced dipole moment in a scattering element $d \tau, o$ is unit vector in the polarization direction of the analyzer, $r$ is a position vector of the scattering element from the center of the spherulite, $k=2 \pi / \lambda$, and $s=s_{0}-s^{\prime}$, and where $\lambda$ is the wavelength of the light within the specimen, $s_{0}$ is the unit vector of the incident ray, and $s^{\prime}$ is the unit vector of the scattered ray.

Stein has formulated the intensity distribution

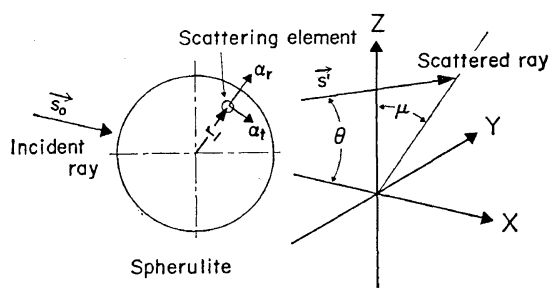

Figure 9. Schematic diagram showing the coordinates system of light-scattering from the spherulite. of polarized light scattered from spherulitic film on the assumptions that the optical anisotropy of the dispersed spherulites in spherical symmetry being given by $\left(\alpha_{r}-\alpha_{t}\right)$, where $\alpha_{r}$ and $\alpha_{t}$ are polarizabilities along the radial and tangential directions of the spherulite dispersed in a uniform medium of polarizability $\alpha_{s}$, and that there is neither the size distribution nor the optical correlation among the dispersed spherulites; i.e., the scattering from an isolated single spherulite of ideally regular texture. The $H_{v}$ scattering intensity distribution has been given by $I=E E^{*}$, where $E^{*}$ is the complex conjugate of $E$, as follows:

$$
\begin{aligned}
I_{H_{v}} & =\frac{C}{h^{6}}\left[\left(\alpha_{r}-\alpha_{t}\right) \cos ^{2}(\theta / 2) \sin \mu \cos \mu\right. \\
& \times(4 \sin U-U \cos U-3 S i U)]^{2}
\end{aligned}
$$

where $C$ is a constant, $\theta$ and $\mu$ are scattering and azimuthal angles, $h=(4 \pi / \lambda) \sin (\theta / 2), U$ is the shape factor given by $(h R), R$ is the radius of the spherulite, and $S i$ is the sine integral.

The intensity distribution predicted from Eq. 2 gives almost circular logarithmic contour plots separated into four hills having maximum intensities at $\theta=\theta_{\max }$ and $\mu=(\pi / 4)+n(\pi / 2)$, where $n=0,1,2,3$, and is very similar to the "four leaf clover pattern" as seen in Figure 5 for specimens such as G 201 and L 402. From Eq. 2, the value of $U$ for the maximum intensity is found as 4.09 , which gives

$$
(4 \pi / \lambda) \sin \left(\theta_{\max } / 2\right) R=4.09
$$

from which the radius of the spherulite can be determined. ${ }^{2}$

In order to modify the above calculation, the following four cases will be taken into consideration: the distribution of size of spherulites, the modification of the spherulite anisotropy so as to vary $\left(\alpha_{r}-\alpha_{t}\right)$ as a function of distance from the center of the spherulite, the modification of the anisotropy at the boundary of the spherulite, and the modification of crystalline texture from a spherulitic to a sheaf-like one.

\section{Distribution of Size of Spherulites}

The formulation has been already demonstrated by Stein on assuming non-correlation between the spherulites to give the following intensity distribution on the basis of additivity of scattered intensities: ${ }^{10}$ 


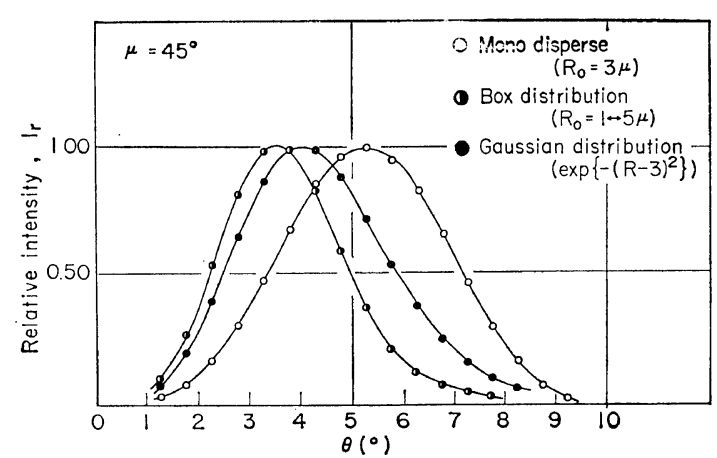

Figure 10. Intensity distribution of $H_{v}$ light-scattering along the scattering angle calculated from Eq. 4 on assuming three types of spherulite size distributions.

$$
\begin{aligned}
I_{H_{v}}= & \int_{R=0}^{\infty} N(R) I_{H_{v}}(R) d R \\
= & B \int_{R=0}^{\infty} \frac{N(R)}{h^{6}}\{4 \sin (h R)-(h R) \cos (h R) \\
& -3 S i(h R)\} d R
\end{aligned}
$$

where $N(R)$ is a distribution function of the radius of spherulites and $B$ is a constant.

Figure 10 shows the results of the calculations from Eq. 4 on assuming the distribution function as monodisperse, box distribution, or Gaussian distribution. As seen in the figure, there is essentially no change of the intensity distribution, except for the shift of $\theta_{\max }$ and some skew of the intensity distribution both to lower scattering angles.

\section{Modifications of Spherulite Anisotropy}

As the distribution of size of the spherulites does not give any essential change for the intensity distribution of scattered light, the modifications of the spherulite anisotropy taking $\left(\alpha_{r}-\alpha_{t}\right)$ as a function of the distance $r$ from the center of the isolated spherulite can be performed in the following two fashions:

$$
\left(\alpha_{r}-\alpha_{t}\right)=\left(\alpha_{r}-\alpha_{t}\right)_{0}(r / R)
$$

and

$$
\left(\alpha_{r}-\alpha_{t}\right)=\left(\alpha_{r}-\alpha_{t}\right)_{0}\left(1-\frac{b r}{R}\right)
$$

where $\left(\alpha_{r}-\alpha_{t}\right)_{0}$ is a constant. Namely, Eq. 5 assumes the increase of the anisotropy from the center (isotropic) to the boundary of the spherulite, while Eq. 6 assumes, in contrast, the decrease of the anisotropy with the parameter $b$ from the center to the boundary.

The amplitudes of scattered lights for the respective cases of Eqs. 5 and 6 may be given by

$$
\begin{aligned}
& E_{H_{v}}=B^{\prime} \frac{1}{h^{3}} \int_{r=0}^{R}\left(\alpha_{r}-\alpha_{t}\right)_{0} \frac{h r}{h R} \\
& \quad \times\left\{3 \cos (h r)+(h r) \sin (h r)-\frac{3 \sin (h r)}{h r}\right\} d(h r)
\end{aligned}
$$

and

$$
\begin{aligned}
& E_{H_{v}}=B^{\prime} \frac{1}{h^{3}} \int_{r=0}^{R}\left(\alpha_{r}-\alpha_{t}\right)_{0} \frac{h R-b h r}{h R} \\
& \quad \times\left\{3 \cos (h r)+(h r) \sin (h r)-\frac{3 \sin (h r)}{h r}\right\} d(h r)
\end{aligned}
$$

where $B^{\prime}$ is a constant.

The intensity distributions of the scattered light from the isolated spherulite may be obtained for the two cases by $I=E \cdot E^{*}$ as follows:

$$
\begin{aligned}
& I_{H_{v}}=D\left\{\left(\alpha_{r}-\alpha_{t}\right)_{0} \cos ^{2}(\theta / 2) \sin \mu \cos \mu\right. \\
& \left.\times \frac{R^{3}}{U^{4}}\left(-U^{2} \cos U+5 U \sin U+8 \cos U-8\right)\right\}^{2}
\end{aligned}
$$

and

$$
\begin{aligned}
& I_{H_{v}}=D\left[\left(\alpha_{r}-\alpha_{t}\right)_{0} \cos ^{2}(\theta / 2) \sin \mu \cos \mu\right. \\
& \quad \times \frac{R^{3}}{U^{3}}\{(4 \sin U-U \cos U-3 S i U) \\
& \left.\left.-\frac{b}{U}\left(-U^{2} \cos U+5 U \sin U+8 \cos U-8\right)\right\}\right]^{2}
\end{aligned}
$$

where $D$ is a constant.

The results of the calculations from neither Eqs. 9 nor 10 essentially change the intensity distribution of the scattered light from that calculated from Eq. 2, except for some shift of the $\theta_{\max }$. Further calculations on assuming $\left(\alpha_{r}-\alpha_{t}\right)$ to be a sort of step function of $r$ or the combination of the distribution of size of the spherulites with the modifications of the spherulite anisotropy also do not give any essential change of the intensity distribution of scattered light. However, 
the fact that the shift of $\theta_{\max }$ in the above calculations for more realistic spherulitic structures than the ideally regular spherulite must be noted in relation to Eq. 3, which has been used for determination of the radius of the spherulite.

\section{Modification of Anisotropy at the Boundary of the Spherulite}

In the Stein formulation as well as in the above two modifications, clear optical discontinuity is assumed at the boundary of the spherulite in order to isolate the spherulites, and, therefore, the calculations of the scattered intensity can be performed on the basis of additivity of the scattered intensity from each spherulite. Here one assumes a more gradual change of anisotropy at the boundary as given by the following representations so that the calculation must be performed on the basis of amplitude additivity of scattered light from the spherulite and the outside of the spherulite:

$$
\begin{aligned}
& R \geqq r \geqq 0 ; \quad\left(\alpha_{r}-\alpha_{t}\right)=\left(\alpha_{r}-\alpha_{t}\right)_{0} \\
& \infty \geqq r \geqq R ; \quad\left(\alpha_{r}-\alpha_{t}\right)=a\left(\alpha_{r}-\alpha_{t}\right)_{0}\left(R^{3} / r^{3}\right)
\end{aligned}
$$

where $a$ is a parameter not only to characterize the unclearness of the boundary but also to take account of the correlation of other spherulites. The functional form of $\left(R^{3} / r^{3}\right)$ to define the parameter $a$ is chosen rather arbitrarily so as to solve the equation easily.

The amplitude of the scattered light may be given by the following equation on the basis of the amplitude additivity:

$$
\begin{aligned}
E_{H_{v}} & =B^{\prime} \frac{1}{h^{3}}\left[\int_{r=0}^{R}\left(\alpha_{r}-\alpha_{t}\right)_{0}\{3 \cos (h r)\right. \\
& \left.+(h r) \sin (h r)-\frac{3 \sin (h r)}{(h r)}\right\} d(h r) \\
& +\int_{r=R}^{\infty} a\left(\alpha_{r}-\alpha_{t}\right)_{0} \frac{R^{3}}{r^{3}} \\
& \left.\times\left\{3 \cos (h r)+(h r) \sin (h r)-\frac{3 \sin (h r)}{(h r)}\right\} d(h r)\right] \\
= & B^{\prime} \frac{R^{3}}{U^{3}}\left(\alpha_{r}-\alpha_{t}\right)_{0}\{(4 \sin U-U \cos U-3 \text { Si } U) \\
& +a(U \cos U-\sin U)\}
\end{aligned}
$$

The scattering intensity may be given by $I=E \cdot E^{*}$ as follows:

$$
\begin{aligned}
I_{H_{v}}= & \frac{G}{h^{6}}\left[\left(\alpha_{r}-\alpha_{t}\right)_{0} \cos ^{2}(\theta / 2) \sin \mu \cos \mu\right. \\
& \times\{(4 \sin U-U \cos U-3 \text { Si } U) \\
& +a(U \operatorname{soc} U-\operatorname{Sin} U)\}]^{2}
\end{aligned}
$$

where $G$ is a constant.

The intensity distribution of scattered light calculated from Eq. 14 is shown in Figures 11 and 12 as the logarithmic contour plot by taking the

$$
\begin{aligned}
& \mathrm{d}=0.1 \\
& \mathrm{R}=4.5 \mu
\end{aligned}
$$

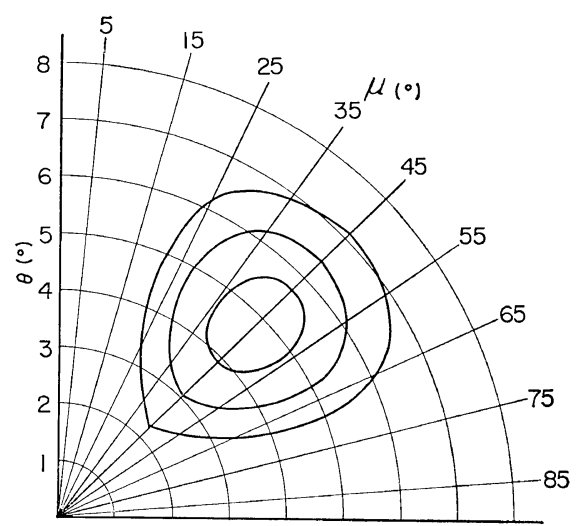

Figure 11. Logarithmic contour plot of $H_{v}$ lightscattering, obtained from Eq. 14 on assuming the radius of the spherulite to be $4.5 \mu$ and the correlation parameter $a$ to be 0.1 .

$$
\begin{aligned}
& a=0.3 \\
& R=4.5 \mu
\end{aligned}
$$

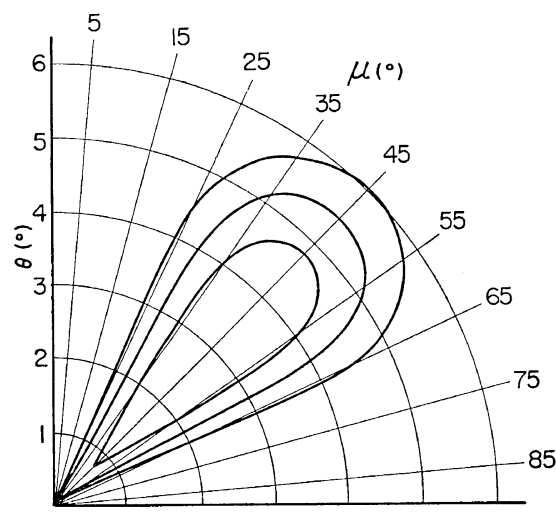

Figure 12. Logarithmic contour plot of $H_{v}$ lightscattering, obtained from Eq. 14 on assuming the radius of the spherulite to be $4.5 \mu$ and the correlation parameter $a$ to be 0.3 . 


\section{Light-Scattering from Polyethylene Spherulite}

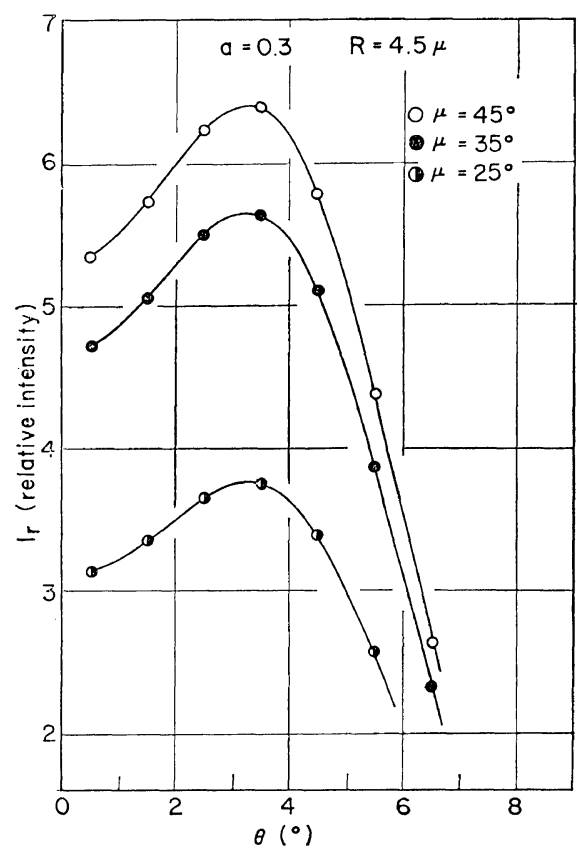

Figure 13. Intensity distributions of $H_{v}$ lightscattering along the scattering angle at particular azimuthal angles of 25,35 , and $45^{\circ}$. (replotted from Figure 12).

parameter $a$ as 0.1 and 0.3 , respectively, and $\left(\alpha_{r}-\alpha_{t}\right)_{0}$ as -0.003 and $R$ as $4.5 \mu$, commonly. As seen in the figures, the change of the intensity distribution to extend the contours to lower scattering angles with increasing of $a$ will be noted. Figure 13 is a re-plot of Figure 12, the intensity distribution along the scattering angle at particular azimuthal angles, $\mu=25$, 35 and $45^{\circ}$.

\section{Modification of Spherulitic Crystalline Texture}

For crystalline polymers of a rather low degree of crystallinity, the crystalline super-structure may be represented by a sheaf-like texture rather than by a spherulitic one. Therefore, a modification of the crystalline texture from spherulitic to sheaflike may give a possibility for realizing the "four tennis rackets" pattern.

For the calculation of the intensity distribution of scattered light, a two-dimensional symmetric sector model of the sheaf-like texture, which is oriented parallel to the surface of the film specimen with random distribution of angle $\delta$, as shown in Figure 14, is taken into consideration. The

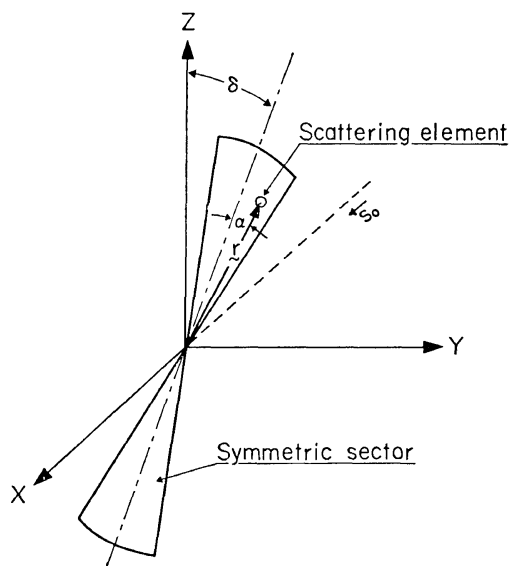

Figure 14. Two-dimensional symmetric sector model of the sheaf-like crystalline texture. The symmetric sectors are assumed to orient parallel to the surface of the film specimen with random distribution of angle $\delta$.

intensity of scattered light from the model of the sheaflike texture may be given by $I=E \cdot E^{*}$, on the basis of additivity of scattered intensity from each sector, as follows:

$$
I=F \int_{\delta=0}^{2 \pi}\left[\int_{\alpha} \int_{r=0}^{R}(M \cdot o) \cos \{k(r \cdot s)\} r d r d \alpha\right]^{2} d \delta
$$

where $F$ is a constant, and $\alpha$ is the angle between a center line of the symmetric sector and a position vector $r$ of a scattering element from the center of the sector.

The $H_{v}$ scattering intensity may be given by

$$
\begin{aligned}
I_{H_{v}}= & F \int_{\hat{\delta}=0}^{2 \pi}\left[\int_{\alpha} \int_{r=0}^{R} A r \cos (B r) d r d \alpha\right]^{2} d \delta \\
= & F \int_{\hat{\delta}=0}^{2 \pi}\left[\left[\int _ { \alpha } \left[\frac{A r}{B} \sin (B R)\right.\right.\right. \\
& \left.\left.+\frac{A}{B^{2}}\{\cos (B R)-1\}\right] d \alpha\right]\left.\right|^{2} d \delta
\end{aligned}
$$

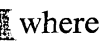

$$
A=\cos (\alpha+\delta) \sin (\alpha+\delta)
$$

and

$$
\begin{aligned}
B= & \frac{2 \pi}{\lambda}\{\sin \theta \sin \mu \sin (\alpha+\delta) \\
& +\sin \theta \cos \mu \cos (\alpha+\delta)\}
\end{aligned}
$$




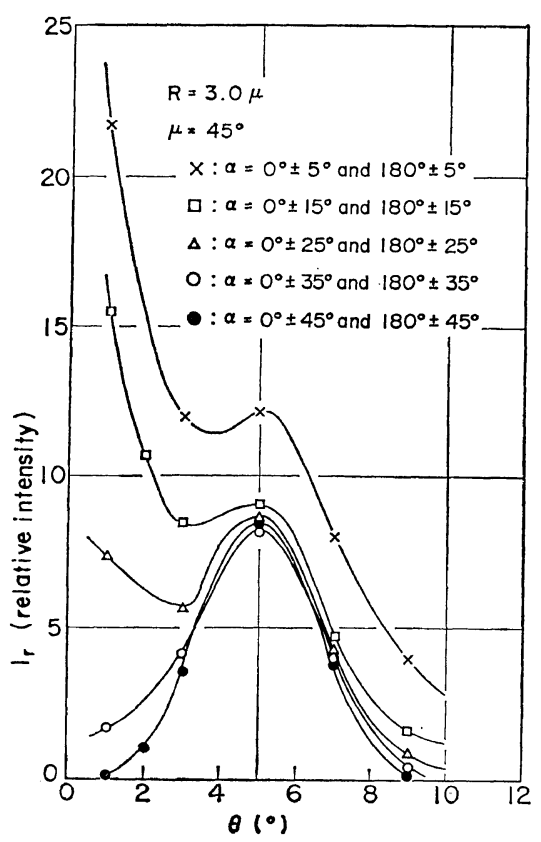

Figure 15. Intensity distribution of $H_{v}$ light-scattering along the scattering angle calculated from Eq. 16 with varying the sector angle $\alpha$ but fixing the values of radius of sector $R$ and azimuthal angle of intensity distribution $\mu$ as 3.0 microns and $45^{\circ}$, respectively.

The results of the numerical calculation of Eq. 16 are plotted against the scattering angle in Figure 15 with varying the sector angle $\alpha$ but fixing the values of $R$ and $\mu$ as 3.0 microns and $45^{\circ}$, respectively. As seen in the figure, the scattered intensity at low scattering angles is remarkably increased by decreasing the sector angle. Although the complete intensity distribution is not demonstrated, ${ }^{*}$ it may be suggested that the more sheaf-like the crystalline texture, the more the $H_{v}$ scattering approaches the "four tennis rackets" pattern, even showing the strongest intensity at the low scattering angles. Such phenomena is often found, as seen in Figure 5, for film specimens, such as YK 50 and KR 1051, having a rather small size of spherulite calculated on the basis of $\theta_{\max }$ in Eq. 3 .

* The complete intensity distribution will be published elsewhere. ${ }^{11}$

\section{CONCLUSIONS}

The $H_{v}$ light-scattering patterns from film specimens molten-cast from different kinds of polyethylenes under identical casting conditions are classified into two groups with good correlation to the spherulite size dependence of some physical indices, such as bulk density and sonic modulus. The light-scattering patterns classified as one group can be represented by the so-called "four leaf clover pattern", well-predicted from Stein's formulation for the isolated spherulite of rather simple and ideally regular anisotropy, while the patterns classified as the remaining group, which appear at wider scattering angles and are less sharp at the boundary of the scattering pattern, have rather long leafstalks and may be called the "four tennis rackets" pattern.

Some modifications of Stein's formulation to realize the intensity distribution of scattered light for the remaining group have been performed by taking the distribution of size of the spherulites into account, taking the spherulite anisotropy, $\left(\alpha_{r}-\alpha_{t}\right)$, as a function of distance from the center of the spherulite, making the boundary of the spherulite less clear, and changing the crystalline texture from a spherulitic to a sheaf-like one. The former two modifications do not essentially change the intensity distribution from that predicted by Stein's formulation, except for some shift of the scattering angle at maximum intensity, while the latter two modifications give the extension of logarithmic contour plot of scattering intensity to low scattering angles, even showing the strongest intensity at low scattering angles for the last modification.

The latter two modifications realize, at least qualitatively, the "four tennis rackets" pattern, suggesting that the imperfection of the spherulite texture, not only in the sense of the texture itself but also in the regularity of its anisotropy, plays an important role. This imperfection of spherulite texture is reflected in the double scattering pattern, which is demonstrated in Figures 7 and 8 for the stretched specimens of YK 3200 and is explained in terms of the existence of at least two types of spherulites differing in mechanical compliance. A more general treatment taking the orientation fluctuation of the principal optical axes of crystalline lamellae within the spherulite 


\section{Light-Scattering from Polyethylene Spherulite}

as a function of distance from the center of spherulite as well as the azimuthal angle of spherulite, may be expected in realizing the "four tennis rackets" pattern. ${ }^{12 *}$

In addition, a combination of Stein's formulation for an isolated spherulite of ideally regular texture with random distribution of rod-shape anisotropic elements, ${ }^{* *}$ may also be expected for realizing the "four tennis rackets" pattern, especially for realizing the strongest intensity at low scattering angles. Furthermore, the asymmetry of spherulitic texture caused by collision of neighbouring spherulites during the growth of spherulitic texture may also be a factor for realizing the "four tennis rackets" pattern. For calculation, however, the fundamental equation of light-scattering represented by Eq. 1, which has been derived on the basis of the symmetric nature of the scattering body, must be modified.

Acknowledgment. The authors are indebted to Professor Richard S. Stein, Polymer Research Institute, University of Massachusetts, Amherst, Mass., U.S.A., who gave valuable comments on this paper.

* The last modification may be understood as one of those extreme cases of the orientation fluctuations of the principal optical axes of crystalline lamellae with respect to the azimuthal angle of the spherulite.

** A formulation of intensity distribution of scattered light from a two dimensional random distribution of rod-shape anisotropic elements has been proposed by Stein et al. ${ }^{13}$ and that from a three dimensional random distribution will be discussed in a successive paper on light scattering from collagen films. ${ }^{14}$
A part of this work was supported by a grant from the Scientific Research Funds (Kagaku Kenkyu-hi, 19214-1967) of the Ministry of Education, Japan.

\section{REFERENCES}

1. P. Debye and A. M. Bueche: J. Appl. Phys., 20, 518 (1949).

2. R. S. Stein and M. B. Rhodes, J. Appl. Phys., 31, 1873 (1960).

3. R. S. Stein and P. R. Wilson, J. Appl. Phys., 33, 1914 (1962).

4. R. S. Stein, P. R. Wilson, and S. N. Stidham, J. Appl. Phys., 34, 46 (1963).

5. I. Harris, J. Polym. Sci., 8, 353 (1952).

6. J. L. Mattews, H. S. Peiser, and R. B. Richards, Acta Cryst., 2, 85 (1949).

7. R. Chiang and P. J. Flory, J. Amer. Chem. Soc., 83, 2857 (1961).

8. S. B. Clough, J. J. van Aartsen, and R. S. Stein, J. Appl. Phys., 36, 3072 (1965).

9. R. J. Samuels, J. Polym. Sci., Part C, 13, 37 (1966); Part A, 3, 1741 (1965).

10. R. S. Stein, S. N. Stidham, and P. R. Wilson, ONR Technical Report, No. 36, Department of Chemistry, University of Massachusetts, Amherst, Mass., 1961.

11. C. Picot, R. S. Stein, M. Motegi, and H. Kawai, to be submitted to J. Polym. Sci., Part A-2.

12. R. S. Stein and W. Chu, private communications.

13. R. S. Stein, P. Erhardt, J. J. van Aartsen, S. Clough, and M. Rhodes, J. Polym. Sci., Part C, 13, 1 (1966).

14. M. Moritani, H. Kawai, and A. Utsuo, to be submitted to this journal. 